DOI: $12737 / 25204$

УДК 674.093

МЕТОДИКА ПОСТАНОВКИ И РЕШЕНИЯ ЗАДАЧИ ОПТИМИЗАЦИИ РАСКРОЯ ПИЛОВОЧНИКА БОЛЬШИХ РАЗМЕРОВ БРУСОВО-РАЗВАЛЬНЫМ СПОСОБОМ С ВЫПИЛИВАНИЕМ ОДНОГО БРУСА И ПЯТИ ПАР БОКОВЫХ ОБРЕЗНЫХ ДОСОК С ПОСЛЕДУЮЩЕЙ РАСПИЛОВКОЙ БРУСА НА ОБРЕЗНЫЕ ДОСКИ

доктор технических наук, профессор А. И. Агапов

ФГБОУ ВО «Вятский государственного университет», г. Киров, Российская Федерация

\begin{abstract}
Впервые составлена математическая модель задачи оптимизации для такой схемы раскроя пиловочника, включая целевую функцию и шесть уравнений связи. В статье рассматривается пифагорическая зона пиловочника. Поэтому целевая функция представлена в виде суммы площадей поперечных сечений обрезных досок. Уравнения связи представляют взаимосвязь диаметра пиловочника в вершинном торце с размерами получаемых обрезных досок. Эта взаимосвязь описывается на основе использования теоремы Пифагора. Такое представление математической модели задачи оптимизации считается классическим. Однако решение такой математической модели классическим методом оказалось проблематичным. Для решения математической модели использовался метод множителей Лагранжа. Предложен алгоритм решения задачи для определения оптимальных размеров брусьев и боковых обрезных досок с учетом ширины пропила. Используя численный метод определены оптимальные размеры брусьев и досок, при которых целевая функция принимает максимальное значение. Оказалось, что с увеличением ширины пропила толщина бруса возрастает, а размеры боковых обрезных досок уменьшаются. Размеры крайних боковых досок с увеличением ширины пропила уменьшаются в большей степени, чем боковые доски, которые расположены ближе к центру бревна. Алгоритм решения задачи оптимизации рекомендуется использовать для расчета и составления поставов при проектировании и эксплуатации лесопильных линий по производству пиломатериалов. При использовании предлагаемого алгоритма решения задачи оптимизации выход пиломатериалов можно увеличить на 3-5 процентов.
\end{abstract}

Ключевые слова: пиловочник, ширина пропила, брус, обрезная доска, математическая модель, целевая функция, уравнения связи, численный метод, алгоритм задачи.

\title{
METHODS OF FORMULATING AND SOLVING OPTIMIZATION PROBLEMS OF CUTTING LOGS OF LARGE SIZE BY BAR-SAWING METHOD WITH CUTTING OUT ONE BAR AND FIVE PAIRS OF SIDE EDGING BOARDS WITH SUBSEQUENT SAWING LUMBER FOR EDGED BOARDS
}

DSc in Engineering, Professor A. I. Agapov

Federal State Budget Education Institution of Higher Professional Education «Vyatka State University», Kirov, Russian Federation

\begin{abstract}
For the first time the mathematical model of task optimization for this scheme of cutting logs, including the objective function and six equations of connection. The article discusses Pythagorean area of the logs. Therefore, the target function is represented as the sum of the cross-sectional areas of edging boards. Equation of the relationship represents the relationship of the diameter of the logs in the vertex end with the size of the resulting edging boards. This relationship is described through the use of the Pythagorean Theorem. Such a representation of the mathematical model of optimization task is considered a classic one. However, the solution of this mathematical model by the classic method is proved to be problematic. For the solution of the mathematical model we used the method of Lagrange multipliers. Solution algorithm to determine the optimal dimensions of the beams and side edging boards taking into account the width of cut is suggested. Using a numerical method, optimal dimensions of the beams and planks are determined, in which the objective function takes the maximum value. It turned out that with the increase of the width of the cut, thickness of the beam increases and the dimensions of the side edging boards reduce. Dimensions of the extreme side planks to increase the width of cut is reduced to a greater extent than the side boards, which are located closer to the center of the log. The algorithm for solving the optimization problem is recommended to use for calculation and preparation of sawing schedule in the design and operation of sawmill lines for timber production. When using the proposed algorithm for solving the optimization problem the output of lumber can be increased to $3-5 \%$.
\end{abstract}

Keywords: saw logs, cut width, beam, edged board, mathematical model, objective function, equation of connection, numerical method, task algorithm. 


\section{Деревопереработка. Химические технологии}

\section{Постановка задачи}

Брусово-развальный способ раскроя пиловочника широко используется в промышленности, так как позволяет за два прохода сформировать обрезные доски. При раскрое пиловочника больших и крупных размеров (диаметр бревна в вершинном торце $60 \ldots 90 \mathrm{~cm}$ ) часто используется схема раскроя с выпиливанием одного бруса и пяти пар боковых обрезных досок (рис. 1) [7, 9].

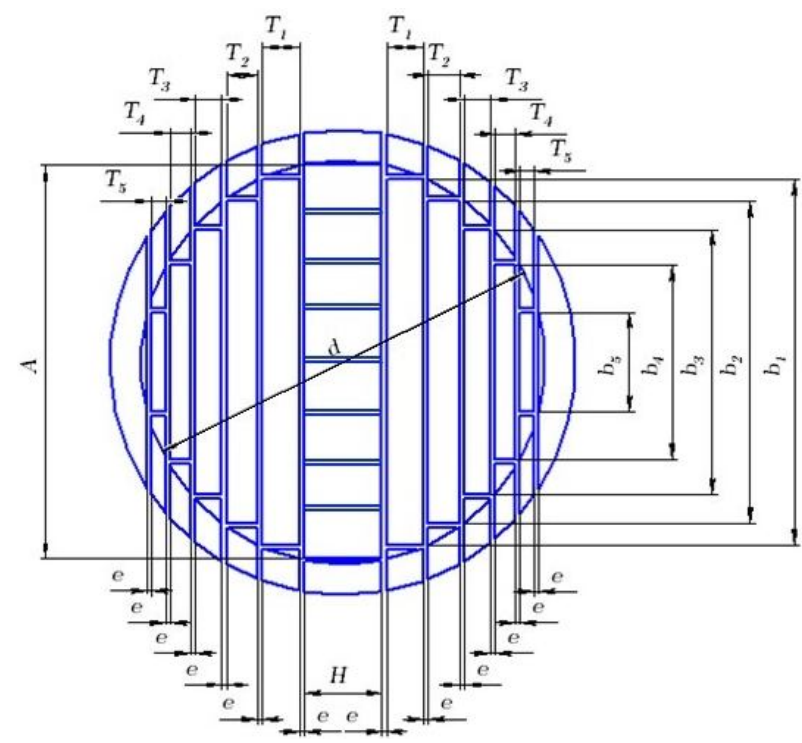

Рис. 1. Схема раскроя пиловочника с выпиливанием одного бруса и пяти пар боковых обрезных досок с учетом ширины пропила и последующей распиловкой бруса на обрезные доски

В теории максимальных поставов обычно определяются оптимальные размеры бруса и досок без учета ширины пропила $[1,6,12]$. Затем при расчете поставов учитываются и ширина пропила и припуск на усушку $[6,8,12,15]$. Такой подход упрощает постановку и решение задачи оптимизации раскроя пиловочника, хотя и вносит определенную погрешность в определение оптимальных размеров пилопродукции. Безусловно ширина пропила повлияет на оптимальные размеры пиломатериалов, но математическая модель при учете ширины пропила усложняется $[2,3]$.

Анализируя ранее выполненные работы по оптимизации раскроя пиловочника [4, 5, 13, 14], можно констатировать, что в этих работах не рассматривалось влияние последующей распиловки бруса на обрезные доски на оптимальные размеры этого бруса и боковых обрезных досок, получаемых при первом проходе раскроя пиловочника. Исследования автора [1] установлено, что при первом проходе раскроя пиловочника брусовым способом с увеличением ширины пропила толщина бруса возрастает, а оптимальные размеры боковых обрезных досок уменьшаются. Однако при последующем раскрое двухкантного бруса (второй проход) будут образовываться потери древесины в опилки, влияние которых на оптимальные размеры бруса и боковых обрезных досок до сих пор не установлено.

Калитеевский Р.Е. $[10,11]$ в своих работах отмечает, что при распиловке с брусовкой наиболее целесообразной толщиной бруса является величина, равная 0,7 $\pm 0,1 \mathrm{~d}$ (где $\mathrm{d}$ - диаметр пиловочника в вершинном торце). Далее в этой работе отмечается, что почти всегда рекомендуется метод последовательной оптимизации - вначале для первого прохода составляют основной постав получения из пифагорической зоны бруса толщиной $(0,6-0,8)$ dи боковых обрезных досок, а затем для второго прохода составляют постав на развал этого бруса. Такой подход упрощает решение задачи оптимизации раскроя пиловочника, но не учитывает взаимосвязь этих операций технологического процесса. В связи с этим была поставлена задача оптимизации с учетом ширины пропила и учета количества пропилов при раскрое бруса на обрезные доски. В таком варианте задача оптимизации раскроя пиловочника поставлена впервые. Для такой схемы раскроя пиловочника пока не определены оптимальные размеры бруса и досок и не определено влияние ширины пропила на оптимальные размеры бруса и боковых обрезных досок и не установлено влияние количества пропилов при раскрое бруса на обрезные доски на оптимальные размеры этого бруса и боковых обрезных досок, получаемых при первом проходе.

\section{Составление математической модели}

Для решения такой задачи составляем математическую модель $[2,5]$. В качестве критерия оптимальности выбираем выход обрезных пиломатериалов, получаемых после первого прохода раскроя пиловочника по данной схеме.Так как в задаче оптимизации раскроя пиловочника рассматривается пифагорическая зона, то, согласно теории макси- 


\section{Деревопереработка. Химические технологии}

мальных поставов [4, 6, 12], длина пиломатериалов, получаемых из пиловочника, равна длине бревна. Поэтому в математической модели длину бревна можно принять за единицу[13, 14]. Тогда целевую функцию представляем в виде суммы площадей поперечных сечений бруса ибоковых обрезных досок

$$
\begin{aligned}
& \mathrm{Z}=\mathrm{HA}+2 \mathrm{~T}_{1} \mathrm{~b}_{1}+2 \mathrm{~T}_{2} \mathrm{~b}_{2}+ \\
& +2 \mathrm{~T}_{3} \mathrm{~b}_{3}+2 \mathrm{~T}_{4} \mathrm{~b}_{4}+2 \mathrm{~T}_{5} \mathrm{~b}_{5}
\end{aligned}
$$

где $\mathrm{H}$ - толщина бруса;

А - ширина пласти бруса;

$\mathrm{T}_{1}, \mathrm{~T}_{2}, \mathrm{~T}_{3}, \mathrm{~T}_{4}, \mathrm{~T}_{5}$ - толщины соответственно первой, второй, третьей, четвертой и пятой пары боковых обрезных досок;

$\mathrm{b}_{1}, \mathrm{~b}_{2}, \mathrm{~b}_{3}, \mathrm{~b}_{4}, \mathrm{~b}_{5}$ - ширины наружной пласти соответственно первой, второй, третьей, четвертой и пятой пары боковых обрезных досок.

Такое представление целевой функции отвечает физической сущности теории максимальных поставов при раскрое пиловочника. В формуле (1) одновременно суммарно учитываются площади поперечных сечений бруса и боковых досок. Можно предположить, что с увеличением объема бруса, объем досок уменьшается и наоборот. Очевидно имеется такое соотношение размеров бруса и досок, при котором объем этой пилопродукции получается максимальным. Такой системный подход отвечает так же требованиям постановки и решения оптимизационных задач.

Уравнения связи представляем в виде зависимостей диаметра пиловочника в вершинном торце с размерами получаемого бруса и боковых досок $[3,5]$. Для этого воспользуемся теоремой Пифагора. Уравнение связи для бруса

$$
\mathrm{d}^{2}-\mathrm{H}^{2}-\mathrm{A}^{2}=0,
$$

где $\mathrm{d}$ - диаметр пиловочника в вершинном торце.

Уравнение связи для первой пары боковых обрезных досок

$$
\begin{gathered}
\mathrm{d}^{2}-\mathrm{b}_{1}^{2}-\left(\mathrm{H}+2 \mathrm{~T}_{1}+2 \mathrm{e}\right)^{2}=0 \\
\mathrm{~d}^{2}-\mathrm{b}_{1}^{2}-\mathrm{H}^{2}-4 \mathrm{~T}_{1}^{2}-4 \mathrm{e}^{2}-4 \mathrm{HT}_{1}-4 \mathrm{He}- \\
-8 \mathrm{~T}_{1} \mathrm{e}=0,
\end{gathered}
$$

где е - ширина пропила.

Уравнение связи для второй пары боковых обрезных досок

$$
\begin{gathered}
\mathrm{d}^{2}-\mathrm{b}_{2}^{2}-\left(\mathrm{H}+2 \mathrm{~T}_{1}+2 \mathrm{~T}_{2}+4 \mathrm{e}\right)^{2}=0, \\
\mathrm{~d}^{2}-\mathrm{b}_{2}^{2}-\mathrm{H}^{2}-4 \mathrm{~T}_{1}^{2}-4 \mathrm{~T}_{2}^{2}-16 \mathrm{e}^{2}-4 \mathrm{HT}_{1}- \\
-4 \mathrm{HT}_{2}-8 \mathrm{He}-8 \mathrm{~T}_{1} \mathrm{~T}_{2}-16 \mathrm{~T}_{1} \mathrm{e}-16 \mathrm{~T}_{2} \mathrm{e}=0 . \quad \text { (4) } \\
\text { Уравнение связи для третьей пары боковых }
\end{gathered}
$$
досок

$$
\begin{array}{r}
\mathrm{d}^{2}-\mathrm{b}_{3}^{2}-\left(\mathrm{H}+2 \mathrm{~T}_{1}+2 \mathrm{~T}_{2}+2 \mathrm{~T}_{3}+6 \mathrm{e}\right)^{2}=0 \\
\mathrm{~d}^{2}-\mathrm{b}_{3}^{2}-\mathrm{H}^{2}-4 \mathrm{~T}_{1}^{2}-4 \mathrm{~T}_{2}^{2}-4 \mathrm{~T}_{3}^{2}-36 \mathrm{e}^{2}- \\
-4 \mathrm{HT}_{1}-4 \mathrm{HT}_{2}-4 \mathrm{HT}_{3}-12 \mathrm{He}-8 \mathrm{~T}_{1} \mathrm{~T}_{2}-8 \mathrm{~T}_{1} \mathrm{~T}_{3}- \\
-8 \mathrm{~T}_{2} \mathrm{~T}_{3}-24 \mathrm{~T}_{1} \mathrm{e}-24 \mathrm{~T}_{2} \mathrm{e}-24 \mathrm{~T}_{3} \mathrm{e}=0 .
\end{array}
$$

Уравнение связи для четвертой пары боковых обрезных досок

$$
\begin{gathered}
\mathrm{d}^{2}-\mathrm{b}_{4}^{2}-\left(\mathrm{H}+2 \mathrm{~T}_{1}+2 \mathrm{~T}_{2}+2 \mathrm{~T}_{3}+2 \mathrm{~T}_{4}+\right. \\
\left.8 \mathrm{e}^{2}\right)=0, \\
\mathrm{~d}^{2}-\mathrm{b}_{4}^{2}-\mathrm{H}^{2}-4 \mathrm{~T}_{1}^{2}-4 \mathrm{~T}_{2}^{2}-4 \mathrm{~T}_{3}^{2}-4 \mathrm{~T}_{4}^{2}-
\end{gathered}
$$$$
64 \mathrm{e}^{2}--4 \mathrm{HT}_{1}-4 \mathrm{HT}_{2}-4 \mathrm{HT}_{3}-4 \mathrm{HT}_{4}-16 \mathrm{He}-
$$$$
8 \mathrm{~T}_{1} \mathrm{~T}_{2}--8 \mathrm{~T}_{1} \mathrm{~T}_{3}-8 \mathrm{~T}_{1} \mathrm{~T}_{4}-8 \mathrm{~T}_{2} \mathrm{~T}_{3}-8 \mathrm{~T}_{2} \mathrm{~T}_{4}-8 \mathrm{~T}_{3} \mathrm{~T}_{4}-
$$$$
-32 \mathrm{~T}_{1} \mathrm{e}-32 \mathrm{~T}_{2} \mathrm{e}-32 \mathrm{~T}_{3} \mathrm{e}-32 \mathrm{~T}_{4} \mathrm{e}=0 \text {. }
$$

Уравнение связи для пятой пары боковых обрезных досок

$$
\begin{gathered}
\mathrm{d}^{2}-\mathrm{b}_{5}^{2}-\left(\mathrm{H}+2 \mathrm{~T}_{1}+2 \mathrm{~T}_{2}+2 \mathrm{~T}_{3}+\right. \\
\left.+2 \mathrm{~T}_{4}+2 \mathrm{~T}_{5}+10 \mathrm{e}\right)^{2}=0 \\
\mathrm{~d}^{2}-\mathrm{b}_{5}^{2}-\mathrm{H}^{2}-4 \mathrm{~T}_{1}^{2}-4 \mathrm{~T}_{2}^{2}-4 \mathrm{~T}_{3}^{2}-4 \mathrm{~T}_{4}^{2}-4 \mathrm{~T}_{5}^{2} \\
-100 \mathrm{e}^{2}-4 \mathrm{HT}_{1}-4 \mathrm{HT}_{2}-4 \mathrm{HT}_{3} \\
-4 \mathrm{HT}_{4}-4 \mathrm{HT}_{5}-20 \mathrm{He}-8 \mathrm{~T}_{1} \mathrm{~T}_{2} \\
-8 \mathrm{~T}_{1} \mathrm{~T}_{3}-8 \mathrm{~T}_{1} \mathrm{~T}_{4}-8 \mathrm{~T}_{1} \mathrm{~T}_{5}-8 \mathrm{~T}_{2} \mathrm{~T}_{3} \\
-8 \mathrm{~T}_{2} \mathrm{~T}_{4}-8 \mathrm{~T}_{2} \mathrm{~T}_{5}-8 \mathrm{~T}_{3} \mathrm{~T}_{4}-8 \mathrm{~T}_{3} \mathrm{~T}_{5}-8 \mathrm{~T}_{4} \mathrm{~T}_{5}- \\
-40 \mathrm{~T}_{1} \mathrm{e}-40 \mathrm{~T}_{2} \mathrm{e}-40 \mathrm{~T}_{3} \mathrm{e}-40 \mathrm{~T}_{4} \mathrm{e}-40 \mathrm{~T}_{5} \mathrm{e}=0 .
\end{gathered}
$$

Для решения задачи воспользуемся методом множителей Лагранжа [4,5]. Функция Лагранжа будет иметь вид

$$
\begin{aligned}
\mathrm{L}=\mathrm{HA} & +2 \mathrm{~T}_{1} \mathrm{~b}_{1}+2 \mathrm{~T}_{2} \mathrm{~b}_{2}+2 \mathrm{~T}_{3} \mathrm{~b}_{3}+ \\
+2 \mathrm{~T}_{4} \mathrm{~b}_{4} & +2 \mathrm{~T}_{5} \mathrm{~b}_{5}+\lambda\left(\mathrm{d}^{2}-\mathrm{H}^{2}-\mathrm{A}^{2}\right) \\
& +\lambda_{1}\left(\mathrm{~d}^{2}-\mathrm{b}_{1}^{2}-\mathrm{H}^{2}-4 \mathrm{e}^{2}-4 \mathrm{HT}_{1}\right. \\
& \left.-4 \mathrm{~T}_{1}^{2}-4 \mathrm{He}-8 \mathrm{~T}_{1} \mathrm{e}\right)+\lambda_{2}\left(\mathrm{~d}^{2}-\mathrm{b}_{2}^{2}\right. \\
& -\mathrm{H}^{2}-4 \mathrm{~T}_{1}^{2}-4 \mathrm{~T}_{2}^{2}-16 \mathrm{e}^{2}-4 \mathrm{HT}_{1} \\
& -4 \mathrm{HT}_{2}-8 \mathrm{~T}_{1} \mathrm{~T}_{2}-8 \mathrm{He}-16 \mathrm{~T}_{1} \mathrm{e} \\
& \left.-16 \mathrm{~T}_{2} \mathrm{e}\right)+\lambda_{3}\left(\mathrm{~d}^{2}-\mathrm{b}_{3}^{2}-\mathrm{H}^{2}-4 \mathrm{~T}_{1}^{2}\right. \\
& -4 \mathrm{~T}_{2}^{2}-4 \mathrm{~T}_{3}^{2}-36 \mathrm{e}^{2}-4 \mathrm{HT}_{1} \\
& -4 \mathrm{HT}_{2}-4 \mathrm{HT}_{3}-8 \mathrm{~T}_{1} \mathrm{~T}_{2}-8 \mathrm{~T}_{1} \mathrm{~T}_{3} \\
& -8 \mathrm{~T}_{2} \mathrm{~T}_{3}-12 \mathrm{He}^{2}-24 \mathrm{~T}_{1} \mathrm{e}-24 \mathrm{~T}_{2} \mathrm{e} \\
& \left.-24 \mathrm{~T}_{3} \mathrm{e}\right)+
\end{aligned}
$$




\section{Деревопереработка. Химические технологии}

$$
\begin{aligned}
+\lambda_{4}\left(\mathrm{~d}^{2}-\mathrm{b}_{4}^{2}-\right. & \mathrm{H}^{2}-4 \mathrm{~T}_{1}^{2}-4 \mathrm{~T}_{2}^{2}-4 \mathrm{~T}_{3}^{2}-4 \mathrm{~T}_{4}^{2}-64 \mathrm{e}^{2} \\
& -4 \mathrm{HT}_{1}-4 \mathrm{HT}_{2}-4 \mathrm{HT}_{3}-4 \mathrm{HT}_{4} \\
& -8 \mathrm{~T}_{1} \mathrm{~T}_{2}-8 \mathrm{~T}_{1} \mathrm{~T}_{3}-8 \mathrm{~T}_{1} \mathrm{~T}_{4}-8 \mathrm{~T}_{2} \mathrm{~T}_{3} \\
& -8 \mathrm{~T}_{2} \mathrm{~T}_{4}-8 \mathrm{~T}_{3} \mathrm{~T}_{4}-16 \mathrm{He}-32 \mathrm{~T}_{1} \mathrm{e} \\
& \left.-32 \mathrm{~T}_{2} \mathrm{e}-32 \mathrm{~T}_{3} \mathrm{e}-32 \mathrm{~T}_{4} \mathrm{e}\right)+\lambda_{5}\left(\mathrm{~d}^{2}\right. \\
& -\mathrm{b}_{5}^{2}-\mathrm{H}^{2}-4 \mathrm{~T}_{1}^{2}-4 \mathrm{~T}_{2}^{2}-4 \mathrm{~T}_{3}^{2} \\
& -4 \mathrm{~T}_{4}^{2}-4 \mathrm{~T}_{5}^{2}-100 \mathrm{e}^{2}-4 \mathrm{HT}_{1} \\
& -4 \mathrm{HT}_{2}-4 \mathrm{HT}_{3}-4 \mathrm{HT}_{4}-4 \mathrm{HT}_{5}
\end{aligned}
$$

$$
\begin{aligned}
& \int \frac{\partial \mathrm{L}}{\partial \mathrm{A}}=\mathrm{H}-2 \lambda \mathrm{A}=0, \\
& \frac{\partial \mathrm{L}}{\partial \mathrm{H}}=\mathrm{A}-2 \lambda \mathrm{H}-2 \lambda_{1} \mathrm{H}-4 \lambda_{1} \mathrm{~T}_{1}-4 \lambda_{1} \mathrm{e}-2 \lambda_{2} \mathrm{H}-4 \lambda_{2} \mathrm{~T}_{1}-4 \lambda_{2} \mathrm{~T}_{2}-8 \lambda_{2} \mathrm{e}-2 \lambda_{3} \mathrm{H}- \\
& -4 \lambda_{3} \mathrm{~T}_{1}-4 \lambda_{3} \mathrm{~T}_{2}-4 \lambda_{3} \mathrm{~T}_{3}-12 \lambda_{3} \mathrm{e}-2 \lambda_{4} \mathrm{H}-4 \lambda_{4} \mathrm{~T}_{1}-4 \lambda_{4} \mathrm{~T}_{2}-4 \lambda_{4} \mathrm{~T}_{3}-4 \lambda_{4} \mathrm{~T}_{4}- \\
& -16 \lambda_{4} \mathrm{e}-4 \lambda_{5} \mathrm{~T}_{5}-2 \lambda_{5} \mathrm{H}-4 \lambda_{5} \mathrm{~T}_{1}-4 \lambda_{5} \mathrm{~T}_{2}-4 \lambda_{5} \mathrm{~T}_{3}-4 \lambda_{5} \mathrm{~T}_{4}-20 \lambda_{5} e=0 \text {, } \\
& \frac{\partial \mathrm{L}}{\partial \mathrm{b}_{1}}=2 \mathrm{~T}_{1}-2 \lambda_{1} \mathrm{~b}_{1}=0, \\
& \frac{\partial \mathrm{L}}{\partial \mathrm{T}_{1}}=2 \mathrm{~b}_{1}-4 \lambda_{1} \mathrm{H}-8 \lambda_{1} \mathrm{~T}_{1}-8 \lambda_{1} \mathrm{e}-4 \lambda_{2} \mathrm{H}-8 \lambda_{2} \mathrm{~T}_{1}-8 \lambda_{2} \mathrm{~T}_{2}-16 \lambda_{2} \mathrm{e}-4 \lambda_{3} \mathrm{H}- \\
& -8 \lambda_{3} \mathrm{~T}_{1}-8 \lambda_{3} \mathrm{~T}_{2}-8 \lambda_{3} \mathrm{~T}_{3}-24 \lambda_{3} \mathrm{e}-4 \lambda_{4} \mathrm{H}-8 \lambda_{4} \mathrm{~T}_{1}-8 \lambda_{4} \mathrm{~T}_{2}-8 \lambda_{4} \mathrm{~T}_{3}-8 \lambda_{4} \mathrm{~T}_{4}- \\
& -32 \lambda_{4} \mathrm{e}-8 \lambda_{5} \mathrm{~T}_{5}-4 \lambda_{5} \mathrm{H}-8 \lambda_{5} \mathrm{~T}_{1}-8 \lambda_{5} \mathrm{~T}_{2}-8 \lambda_{5} \mathrm{~T}_{3}-8 \lambda_{5} \mathrm{~T}_{4}-40 \lambda_{5} e=0 \text {, } \\
& \frac{\partial \mathrm{L}}{\partial \mathrm{b}_{2}}=2 \mathrm{~T}_{2}-2 \lambda_{2} \mathrm{~b}_{2}=0, \\
& \frac{\partial \mathrm{L}}{\partial \mathrm{T}_{2}}=2 \mathrm{~b}_{2}-4 \lambda_{2} \mathrm{H}-8 \lambda_{2} \mathrm{~T}_{2}-8 \lambda_{2} \mathrm{~T}_{1}-16 \lambda_{2} \mathrm{e}-8 \lambda_{3} \mathrm{~T}_{2}-4 \lambda_{3} \mathrm{H}-8 \lambda_{3} \mathrm{~T}_{1}- \\
& -8 \lambda_{3} \mathrm{~T}_{3}-24 \lambda_{3} \mathrm{e}-4 \lambda_{4} \mathrm{H}-8 \lambda_{4} \mathrm{~T}_{1}-8 \lambda_{4} \mathrm{~T}_{2}-8 \lambda_{4} \mathrm{~T}_{3}-8 \lambda_{4} \mathrm{~T}_{4}-32 \lambda_{4} \mathrm{e}- \\
& -8 \lambda_{5} \mathrm{~T}_{5}-4 \lambda_{5} \mathrm{H}-8 \lambda_{5} \mathrm{~T}_{1}-8 \lambda_{5} \mathrm{~T}_{2}-8 \lambda_{5} \mathrm{~T}_{3}-8 \lambda_{5} \mathrm{~T}_{4}-40 \lambda_{5} e=0 \text {, } \\
& \frac{\partial \mathrm{L}}{\partial \mathrm{b}_{3}}=2 \mathrm{~T}_{3}-2 \lambda_{3} \mathrm{~b}_{3}=0, \\
& \frac{\partial \mathrm{L}}{\partial \mathrm{T}_{3}}=2 \mathrm{~b}_{3}-4 \lambda_{3} \mathrm{H}-8 \lambda_{3} \mathrm{~T}_{3}-8 \lambda_{3} \mathrm{~T}_{1}-8 \lambda_{3} \mathrm{~T}_{2}-24 \lambda_{3} \mathrm{e}-8 \lambda_{4} \mathrm{~T}_{3}-4 \lambda_{4} \mathrm{H}- \\
& -8 \lambda_{4} \mathrm{~T}_{1}-8 \lambda_{4} \mathrm{~T}_{2}-8 \lambda_{4} \mathrm{~T}_{4}-32 \lambda_{4} \mathrm{e}- \\
& -8 \lambda_{5} \mathrm{~T}_{5}-4 \lambda_{5} \mathrm{H}-8 \lambda_{5} \mathrm{~T}_{1}-8 \lambda_{5} \mathrm{~T}_{2}-8 \lambda_{5} \mathrm{~T}_{3}-8 \lambda_{5} \mathrm{~T}_{4}-40 \lambda_{5} e=0 \text {, } \\
& \frac{\partial \mathrm{L}}{\partial \mathrm{b}_{4}}=2 \mathrm{~T}_{4}-2 \lambda_{4} \mathrm{~b}_{4}=0, \\
& \frac{\partial \mathrm{L}}{\partial \mathrm{T}_{4}}=2 \mathrm{~b}_{4}-4 \lambda_{4} \mathrm{H}-8 \lambda_{4} \mathrm{~T}_{4}-8 \lambda_{4} \mathrm{~T}_{1}-8 \lambda_{4} \mathrm{~T}_{2}-8 \lambda_{4} \mathrm{~T}_{3}-32 \lambda_{4} \mathrm{e}-8 \lambda_{5} \mathrm{~T}_{5}- \\
& -4 \lambda_{5} \mathrm{H}-8 \lambda_{5} \mathrm{~T}_{1}-8 \lambda_{5} \mathrm{~T}_{2}-8 \lambda_{5} \mathrm{~T}_{3}-8 \lambda_{5} \mathrm{~T}_{4}-40 \lambda_{5} e=0, \\
& \frac{\partial \mathrm{L}}{\partial \mathrm{b}_{5}}=2 \mathrm{~T}_{5}-2 \lambda_{5} \mathrm{~b}_{5}=0, \\
& \frac{\partial \mathrm{L}}{\partial \mathrm{T}_{5}}=2 \mathrm{~b}_{5}-4 \lambda_{5} \mathrm{H}-8 \lambda_{5} \mathrm{~T}_{5}-8 \lambda_{5} \mathrm{~T}_{1}-8 \lambda_{5} \mathrm{~T}_{2}-8 \lambda_{5} \mathrm{~T}_{3}-8 \lambda_{5} \mathrm{~T}_{4}-40 \lambda_{4} \mathrm{e}=0 .
\end{aligned}
$$

Решаем полученную систему уравнений (9) совместно с уравнениями связи. Из предпоследнего уравнения системы (9) находим

$$
\mathrm{T}_{5}=\lambda_{5} \mathrm{~b}_{5}, \quad \lambda_{5}=\frac{\mathrm{T}_{5}}{\mathrm{~b}_{5}} .
$$

Рассматриваем восьмое уравнение системы (9), которое представляем в следующем виде

$$
b_{5}=2 \lambda_{5} \mathrm{H}+4 \lambda_{5} \mathrm{~T}_{1}+4 \lambda_{5} \mathrm{~T}_{2}+4 \lambda_{5} \mathrm{~T}_{3}+
$$

$$
+4 \lambda_{5} \mathrm{~T}_{4}+4 \lambda_{5} \mathrm{~T}_{5}+20 \lambda_{5} \mathrm{e} .
$$

В последнее равенство (11) подставим выражение (10), получим

$$
\begin{gathered}
\mathrm{b}_{5}^{2}=2 \mathrm{~T}_{5}\left(\mathrm{H}+2 \mathrm{~T}_{1}+2 \mathrm{~T}_{2}+2 \mathrm{~T}_{3}+\right. \\
\left.+2 \mathrm{~T}_{4}+2 \mathrm{~T}_{5}+10 \mathrm{e}\right) .
\end{gathered}
$$

Полученное равенство (12) подставляем в уравнение связи (7), получим

Находим частные производные от функции

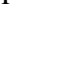

$$
\begin{aligned}
& -8 \mathrm{~T}_{1} \mathrm{~T}_{2}-8 \mathrm{~T}_{1} \mathrm{~T}_{3}-8 \mathrm{~T}_{1} \mathrm{~T}_{4}-8 \mathrm{~T}_{1} \mathrm{~T}_{5} \\
& -8 \mathrm{~T}_{2} \mathrm{~T}_{3}-8 \mathrm{~T}_{2} \mathrm{~T}_{4}-8 \mathrm{~T}_{2} \mathrm{~T}_{5}-8 \mathrm{~T}_{3} \mathrm{~T}_{4} \\
& -8 \mathrm{~T}_{3} \mathrm{~T}_{5}-8 \mathrm{~T}_{4} \mathrm{~T}_{5}-20 \mathrm{He}-40 \mathrm{~T}_{1} \mathrm{e} \\
& -40 \mathrm{~T}_{2} \mathrm{e}-40 \mathrm{~T}_{3} \mathrm{e}-40 \mathrm{~T}_{4} \mathrm{e} \\
& \left.-40 \mathrm{~T}_{5} \mathrm{e}\right)
\end{aligned}
$$

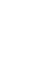

. 


$$
\begin{gathered}
8 \mathrm{~T}_{5}^{2}+6 \mathrm{~T}_{5}\left(\mathrm{H}+2 \mathrm{~T}_{1}+2 \mathrm{~T}_{2}+2 \mathrm{~T}_{3}+2 \mathrm{~T}_{4}+2 \mathrm{~T}_{5}+10 \mathrm{e}\right) \\
+ \\
+\left(\mathrm{H}+2 \mathrm{~T}_{1}+2 \mathrm{~T}_{2}+2 \mathrm{~T}_{3}+2 \mathrm{~T}_{4}+2 \mathrm{~T}_{5}+10 \mathrm{e}\right)^{2}- \\
-\mathrm{d}^{2}=0 .
\end{gathered}
$$

Решая полученное квадратное уравнение (13), получим

$$
\begin{gathered}
\mathrm{T}_{5}= \\
0,125\left(\sqrt{8 \mathrm{~d}^{2}+\left(\mathrm{H}+2 \mathrm{~T}_{1}+2 \mathrm{~T}_{2}+2 \mathrm{~T}_{3}+2 \mathrm{~T}_{4}+10 \mathrm{e}\right)^{2}}-\right. \\
\left.-3\left(\mathrm{H}+2 \mathrm{~T}_{1}+2 \mathrm{~T}_{2}+2 \mathrm{~T}_{3}+2 \mathrm{~T}_{4}+10 \mathrm{e}\right)\right) .
\end{gathered}
$$

Используя уравнение связи (7) толщину пятой пары боковых обрезных досок можно определить по формуле

$$
\begin{aligned}
\mathrm{T}_{5}= & 0,5 \cdot\left(\sqrt{\mathrm{d}^{2}-\mathrm{b}_{5}^{2}}-\left(\mathrm{H}+2 \mathrm{~T}_{1}+2 \mathrm{~T}_{2}+\right.\right. \\
& \left.\left.2 \mathrm{~T}_{3}++2 \mathrm{~T}_{4}+10 \mathrm{e}\right)\right) .
\end{aligned}
$$

Рассматриваем девятое уравнение системы (9), из которого находим

$$
\mathrm{T}_{4}=\lambda_{4} \mathrm{~b}_{4}, \quad \lambda_{4}=\frac{\mathrm{T}_{4}}{\mathrm{~b}_{4}} .
$$

Рассматриваем восьмое уравнение системы (9), которое представляем в следующем виде

$$
\begin{gathered}
\mathrm{b}_{4}=2 \lambda_{4} \mathrm{H}+4 \lambda_{4} \mathrm{~T}_{1}+4 \lambda_{4} \mathrm{~T}_{2}+4 \lambda_{4} \mathrm{~T}_{3}+ \\
4 \lambda_{4} \mathrm{~T}_{4}++16 \lambda_{4} \mathrm{e}+\mathrm{b}_{5} .
\end{gathered}
$$

В последнее равенство (16) подставим выражение (15), получим

$$
\begin{gathered}
\mathrm{b}_{4}^{2}=2 \mathrm{~T}_{4}\left(\mathrm{H}+2 \mathrm{~T}_{1}+2 \mathrm{~T}_{2}+2 \mathrm{~T}_{3}+2 \mathrm{~T}_{4}+8 \mathrm{e}\right)+ \\
+\mathrm{b}_{4} \mathrm{~b}_{5} .
\end{gathered}
$$

Из последнего равенства (17) можно определить ширину пятой пары боковыхобрезных досок

$$
\begin{gathered}
\mathrm{b}_{5}=\mathrm{b}_{4}-\frac{2 \mathrm{~T}_{4}}{\mathrm{~b}_{4}}\left(\mathrm{H}+2 \mathrm{~T}_{1}+2 \mathrm{~T}_{2}+2 \mathrm{~T}_{3}+2 \mathrm{~T}_{4}+\right. \\
+8 \mathrm{e}) .
\end{gathered}
$$

Рассматриваем седьмое уравнение системы (9), из которого находим

$$
\mathrm{T}_{3}=\lambda_{3} \mathrm{~b}_{3}, \quad \lambda_{3}=\frac{\mathrm{T}_{3}}{\mathrm{~b}_{3}} .
$$

Рассматриваем восьмое уравнение системы (9), которое представляем в следующем виде

$$
\begin{gathered}
\mathrm{b}_{3}=2 \lambda_{3} \mathrm{H}+4 \lambda_{3} \mathrm{~T}_{1}+4 \lambda_{3} \mathrm{~T}_{2}+4 \lambda_{3} \mathrm{~T}_{3}+ \\
+12 \lambda_{3} \mathrm{e}+\mathrm{b}_{4} .
\end{gathered}
$$

В последнее равенство (20) подставим выражение (19), получим

$$
\begin{aligned}
\mathrm{b}_{3}^{2}=2 \mathrm{HT}_{3}+ & 4 \mathrm{~T}_{1} \mathrm{~T}_{3}+4 \mathrm{~T}_{2} \mathrm{~T}_{3}+4 \mathrm{~T}_{3}^{2}+12 \mathrm{~T}_{3} \mathrm{e}+ \\
& +\mathrm{b}_{3} \mathrm{~b}_{4} .
\end{aligned}
$$

Из последнего равенства (21) можно определить ширину четвертой пары боковых обрезных досок

$$
\begin{gathered}
\mathrm{b}_{4}=\mathrm{b}_{3}-\frac{2 \mathrm{~T}_{3}}{\mathrm{~b}_{3}}\left(\mathrm{H}+2 \mathrm{~T}_{1}+2 \mathrm{~T}_{2}++2 \mathrm{~T}_{3}+\right. \\
+6 \mathrm{e}) .
\end{gathered}
$$

Используя уравнение связи (6) и зная ширину четвертой пары боковых досок, можно определить толщину этой пары боковых досок

$$
\begin{gathered}
\mathrm{T}_{4}=0,5 \cdot\left(\sqrt{\mathrm{d}^{2}-\mathrm{b}_{4}^{2}}-\left(\mathrm{H}+2 \mathrm{~T}_{1}+2 \mathrm{~T}_{2}+2 \mathrm{~T}_{3}+\right.\right. \\
+8 \mathrm{e})) .
\end{gathered}
$$

Рассматриваем пятое уравнение системы (9), получим

$$
\mathrm{T}_{2}=\lambda_{2} \mathrm{~b}_{2}, \quad \lambda_{2}=\frac{\mathrm{T}_{2}}{\mathrm{~b}_{2}} .
$$

Рассматриваем шестое уравнение системы (9),которое с учетом уравнения (20) можно представить в следующем виде

$$
\mathrm{b}_{2}=4 \lambda_{2} \mathrm{~T}_{2}+2 \lambda_{2} \mathrm{H}+4 \lambda_{2} \mathrm{~T}_{1}+8 \lambda_{2} \mathrm{e}+\mathrm{b}_{3} .(25)
$$

В последнее равенство (25) подставим выражение (24), получим

$$
\mathrm{b}_{2}^{2}=4 \mathrm{~T}_{2}^{2}+2 \mathrm{HT}_{2}+4 \mathrm{~T}_{1} \mathrm{~T}_{2}+8 \mathrm{~T}_{2} \mathrm{e}+\mathrm{b}_{2} \mathrm{~b}_{3} \text {. }
$$

Из последнего равенства (26) можно выразить ширину третьей пары боковыхобрезных досок

$$
\mathrm{b}_{3}=\mathrm{b}_{2}-\frac{2 \mathrm{~T}_{2}}{\mathrm{~b}_{2}}\left(\mathrm{H}+2 \mathrm{~T}_{1}+2 \mathrm{~T}_{2}+4 \mathrm{e}\right) \text {. }
$$

Используя уравнение связи (5) и зная ширину третьей пары боковыхобрезных досок, можно определить толщину этой третьей пары боковых обрезных досок

$$
\mathrm{T}_{3}=0,5\left(\sqrt{\mathrm{d}^{2}-\mathrm{b}_{3}^{2}}-\left(\mathrm{H}+2 \mathrm{~T}_{1}+2 \mathrm{~T}_{2}+6 \mathrm{e}\right)\right) \cdot(28)
$$

Рассматриваем третье уравнение системы (9), из которого находим

$$
\mathrm{T}_{1}=\lambda_{1} \mathrm{~b}_{1}, \quad \lambda_{1}=\frac{\mathrm{T}_{1}}{\mathrm{~b}_{1}} .
$$

Рассматриваем четвертое уравнение системы (9), которое с учетом уравнения (25) можно представить в следующем виде

$$
\mathrm{b}_{1}=2 \lambda_{1} \mathrm{H}+4 \lambda_{1} \mathrm{~T}_{1}+4 \lambda_{1} \mathrm{e}+\mathrm{b}_{2} .
$$

В последнее равенство (30) подставляем выражение (29), получим

$$
\mathrm{b}_{1}^{2}=2 \mathrm{HT}_{1}+4 \mathrm{~T}_{1}^{2}+4 \mathrm{~T}_{1} \mathrm{e}+\mathrm{b}_{1} \mathrm{~b}_{2} .
$$

Из последнего равенства (31) определяем ширину наружной пласти второй пары боковых обрезных досок

$$
\mathrm{b}_{2}=\mathrm{b}_{1}-\left(\frac{2 \mathrm{~T}_{1}}{\mathrm{~b}_{1}}\right) \cdot\left(\mathrm{H}+2 \mathrm{~T}_{1}+2 \mathrm{e}\right) .
$$

Зная ширину второй пары боковых обрезных досок, толщину этих досок можно определить из уравнения связи (4) 
$\mathrm{T}_{2}=0,5\left(\sqrt{\mathrm{d}^{2}-\mathrm{b}_{2}^{2}}-\left(\mathrm{H}+2 \mathrm{~T}_{1}+4 \mathrm{e}\right)\right)$.

Рассматриваем первое уравнение системы(9), которое представляем в виде

$$
\mathrm{H}=2 \lambda \mathrm{A}, \quad \lambda=\frac{\mathrm{H}}{2 \mathrm{~A}} .
$$

Рассматриваем второе уравнение системы(9), которое с учетом четвертого уравнения этой системы можно записать в следующем виде:

$$
\mathrm{A}=2 \lambda \mathrm{H}+\mathrm{b}_{1} \text {. }
$$

В последнее равенство(35) подставляем выражение(34), получим

$$
\mathrm{A}^{2}=\mathrm{H}^{2}+\mathrm{Ab}_{1}
$$

Из уравнения связи (2) можно написать

$$
\mathrm{A}^{2}=\mathrm{d}^{2}-\mathrm{H}^{2} \text {. }
$$

Используя равенство (37), выражение (36) можно представить в следующем виде:

$$
\mathrm{d}^{2}-2 \mathrm{H}^{2}=\mathrm{Ab}_{1} \text {. }
$$

Из последнего равенства (38) можно определить ширину первой пары боковых обрезных досок

$$
\mathrm{b}_{1}=\frac{\mathrm{d}^{2}-2 \mathrm{H}^{2}}{\mathrm{~A}}
$$

Зная ширину первой пары боковых обрезных досок, из уравнения связи (3) можно определить толщину этих досок

$$
\mathrm{T}_{1}=0,5\left(\sqrt{\mathrm{d}^{2}-\mathrm{b}_{1}^{2}}-(\mathrm{H}+2 \mathrm{e})\right) .
$$

Если в формулу (40) подставить уравнение (39), а затем решить его, то получим

$$
\mathrm{T}_{1}=0,5\left(\mathrm{H} \sqrt{\frac{3 \mathrm{~d}^{2}-4 \mathrm{H}^{2}}{\mathrm{~d}^{2}-\mathrm{H}^{2}}}-(\mathrm{H}+2 \mathrm{e})\right) .
$$

Таким образом, рассмотрены все уравнения системы (9), и учтены все уравнения связи. Получены формулы для определения оптимальных размеров бруса и досок, но по которым непосредственно невозможно рассчитать оптимальные размерыбруса и досок.

\section{Алгоритм решения задачи}

Для решения задачи воспользуемся численным методом. Задаемся толщиной бруса, а остальные размеры бруса и досок, а также величину целевой функции определяем по формулам. С целью упрощения расчетов и анализа результатоввсе полученные ранее формулы представляем в относительных единицах, полагая $\mathrm{m}_{\mathrm{H}}=\mathrm{H} / \mathrm{d}$. Тогда алгоритм решения задачи будет иметь следующий вид [5].

Относительная ширина пласти бруса $[1,5]$

$$
\mathrm{m}_{\mathrm{A}}=\frac{\mathrm{A}}{\mathrm{d}}=\sqrt{1-\mathrm{m}_{\mathrm{H}}^{2}} \text {. }
$$

Относительная ширина первой пары боковых обрезных досок

$$
\mathrm{m}_{\mathrm{b}_{1}}=\frac{\mathrm{b}_{1}}{\mathrm{~d}}=\frac{1-2 \mathrm{~m}_{\mathrm{H}}^{2}}{\mathrm{~m}_{\mathrm{A}}} .
$$

Относительная толщина первой пары боковых обрезных досок

$$
0,5 \cdot\left(\sqrt{1-\mathrm{m}_{\mathrm{b}_{1}}^{2}}-\left(\mathrm{m}_{\mathrm{H}}++2 \mathrm{~m}_{\mathrm{e}}\right)\right)
$$$$
\mathrm{m}_{\mathrm{T}_{1}}=\frac{\mathrm{T}_{1}}{\mathrm{~d}}=
$$

Относительная ширина наружной пласти второй пары боковых обрезных досок

$$
\mathrm{m}_{\mathrm{b}_{2}}=\frac{\mathrm{b}_{2}}{\mathrm{~d}}=\mathrm{m}_{\mathrm{b}_{1}}-\left(\frac{2 \mathrm{~m}_{\mathrm{T}_{1}}}{\mathrm{~m}_{\mathrm{b}_{1}}}\right) \cdot\left(\mathrm{m}_{\mathrm{H}}+2 \mathrm{~m}_{\mathrm{T}_{1}}+2 \mathrm{~m}_{\mathrm{e}}\right)(45)
$$

Относительная толщина второй пары боковых обрезных досок

$$
\begin{gathered}
\mathrm{m}_{\mathrm{T}_{2}}=\frac{\mathrm{T}_{2}}{\mathrm{~d}}=0,5 \cdot\left(\sqrt{1-\mathrm{m}_{\mathrm{b}_{2}}^{2}}-\left(\mathrm{m}_{\mathrm{H}}+2 \mathrm{~m}_{\mathrm{T}_{1}}+\right.\right. \\
\left.\left.+4 \mathrm{~m}_{\mathrm{e}}\right)\right) .
\end{gathered}
$$

Относительная ширина наружной пласти третьей пары боковых обрезных досок

$$
\begin{aligned}
\mathrm{m}_{\mathrm{b}_{3}}=\frac{\mathrm{b}_{3}}{\mathrm{~d}}= & \mathrm{m}_{\mathrm{b}_{2}}-\left(\frac{2 \mathrm{~m}_{\mathrm{T}_{2}}}{\mathrm{~m}_{\mathrm{b}_{2}}}\right) \cdot\left(\mathrm{m}_{\mathrm{H}}+2 \mathrm{~m}_{\mathrm{T}_{1}}+\right. \\
& \left.+2 \mathrm{~m}_{\mathrm{T}_{2}}+4 \mathrm{~m}_{\mathrm{e}}\right) .
\end{aligned}
$$

Относительная толщина третьей пары боковых обрезных досок

$$
\begin{aligned}
\mathrm{m}_{\mathrm{T}_{3}}=\frac{\mathrm{T}_{3}}{\mathrm{~d}}=0,5 \cdot( & \sqrt{1-\mathrm{m}_{\mathrm{b}_{3}}^{2}}-\left(\mathrm{m}_{\mathrm{H}}+2 \mathrm{~m}_{\mathrm{T}_{1}}+\right. \\
& \left.\left.+2 \mathrm{~m}_{\mathrm{T}_{2}}+6 \mathrm{~m}_{\mathrm{e}}\right)\right)
\end{aligned}
$$

Относительная ширина наружной пласти четвертой пары боковых обрезных досок

$$
\begin{aligned}
\mathrm{m}_{\mathrm{b}_{4}} & =\frac{\mathrm{b}_{4}}{\mathrm{~d}}=\mathrm{m}_{\mathrm{b}_{3}}-\left(\frac{2 \mathrm{~m}_{\mathrm{T}_{3}}}{\mathrm{~m}_{\mathrm{b}_{3}}}\right) \cdot\left(\mathrm{m}_{\mathrm{H}}+2 \mathrm{~m}_{\mathrm{T}_{1}}+\right. \\
& \left.+2 \mathrm{~m}_{\mathrm{T}_{2}}+2 \mathrm{~m}_{\mathrm{T}_{3}}+6 \mathrm{~m}_{\mathrm{e}}\right) .
\end{aligned}
$$

Относительная толщина четвертой пары боковых обрезных досок

$$
\begin{aligned}
\mathrm{m}_{\mathrm{T}_{4}}=\frac{\mathrm{T}_{4}}{\mathrm{~d}} & =0,5 \cdot\left(\sqrt{1-\mathrm{m}_{\mathrm{b}_{4}}^{2}}-\left(\mathrm{m}_{\mathrm{H}}+2 \mathrm{~m}_{\mathrm{T}_{1}}+\right.\right. \\
& \left.\left.+2 \mathrm{~m}_{\mathrm{T}_{2}}+2 \mathrm{~m}_{\mathrm{T}_{3}}+8 \mathrm{~m}_{\mathrm{e}}\right)\right)
\end{aligned}
$$

Относительная толщина пятой пары боковых обрезных досок

$$
\begin{aligned}
& \mathrm{m}_{\mathrm{T}_{5}} \\
& =0,125 \\
& \cdot \sqrt{8+\left(\mathrm{m}_{\mathrm{H}}+2 \mathrm{~m}_{\mathrm{T}_{1}}+2 \mathrm{~m}_{\mathrm{T}_{2}}+2 \mathrm{~m}_{\mathrm{T}_{3}}+2 \mathrm{~m}_{\mathrm{T}_{4}}+10 \mathrm{~m}_{\mathrm{e}}\right)^{2}} \\
& \left.-3\left(\mathrm{~m}_{\mathrm{H}}+2 \mathrm{~m}_{\mathrm{T}_{1}}+2 \mathrm{~m}_{\mathrm{T}_{2}}+2 \mathrm{~m}_{\mathrm{T}_{3}}+2 \mathrm{~m}_{\mathrm{T}_{4}}+10 \mathrm{~m}_{\mathrm{e}}\right)\right) .
\end{aligned}
$$


Относительная толщина пятой пары боковых обрезных досок

$$
\begin{gathered}
\mathrm{m}_{\mathrm{T}_{5}}=0,5 \cdot\left(\sqrt{1-\mathrm{m}_{\mathrm{b}_{5}}^{2}}-\left(\mathrm{m}_{\mathrm{H}}+2 \mathrm{~m}_{\mathrm{T}_{1}}+\right.\right. \\
\left.\left.2 \mathrm{~m}_{\mathrm{T}_{2}}++2 \mathrm{~m}_{\mathrm{T}_{3}}+2 \mathrm{~m}_{\mathrm{T}_{4}}+10 \mathrm{~m}_{\mathrm{e}}\right)\right) .
\end{gathered}
$$

Относительная ширина наружной пласти пятой пары боковых обрезных досок

$$
\begin{aligned}
\mathrm{m}_{\mathrm{b}_{5}} & =2 \mathrm{~m}_{\mathrm{T}_{5}}\left(\mathrm{~m}_{\mathrm{H}}+2 \mathrm{~m}_{\mathrm{T}_{1}}+2 \mathrm{~m}_{\mathrm{T}_{2}}+2 \mathrm{~m}_{\mathrm{T}_{3}}+\right. \\
& \left.+2 \mathrm{~m}_{\mathrm{T}_{4}}+2 \mathrm{~m}_{\mathrm{T}_{5}}+10 \mathrm{~m}_{\mathrm{e}}\right) .
\end{aligned}
$$

Относительная ширина наружной пласти пятой пары боковых обрезных досок

$$
\begin{gathered}
\mathrm{m}_{\mathrm{b}_{5}}=\mathrm{m}_{\mathrm{b}_{4}}-\left(\frac{2 \mathrm{~m}_{\mathrm{T}_{4}}}{\mathrm{~m}_{\mathrm{b}_{4}}}\right) \cdot\left(\mathrm{m}_{\mathrm{H}}+2 \mathrm{~m}_{\mathrm{T}_{1}}+\right. \\
\left.2 \mathrm{~m}_{\mathrm{T}_{2}}++2 \mathrm{~m}_{\mathrm{T}_{3}}+2 \mathrm{~m}_{\mathrm{T}_{4}}+8 \mathrm{~m}_{\mathrm{e}}\right) .
\end{gathered}
$$

Относительная площадь поперечного сечения первой пары боковых обрезных досок

$$
\mathrm{Z}_{\text {д1 }}=2 \mathrm{~m}_{\mathrm{b} 1} \times \mathrm{m}_{\mathrm{T} 1} .
$$

Относительная площадь поперечного сечения второй пары боковых обрезных досок

$$
\mathrm{Z}_{\text {д2 }}=2 \mathrm{~m}_{\mathrm{b} 2} \times \mathrm{m}_{\mathrm{T} 2} .
$$

Относительная площадь поперечного сечения третьей пары боковых обрезных досок

$$
\mathrm{Z}_{\text {д3 }}=2 \mathrm{~m}_{\mathrm{b} 3} \times \mathrm{m}_{\mathrm{T} 3} .
$$

Относительная площадь поперечного сечения четвертой пары боковых обрезных досок

$$
\mathrm{Z}_{\text {д4 }_{4}}=2 \mathrm{~m}_{\mathrm{b}_{4}} \times \mathrm{m}_{\mathrm{T}_{4}} .
$$

Относительная площадь поперечного сечения пятой пары боковых обрезных досок

$$
\mathrm{Z}_{\text {म5 }}=2 \mathrm{~m}_{\mathrm{b}_{5}} \times \mathrm{m}_{\mathrm{T}_{5}} .
$$

Суммарная относительная площадь поперечного сечения боковых обрезных досок

$$
\mathrm{Z}_{\text {д }}=\mathrm{Z}_{\text {д1 }}+\mathrm{Z}_{\text {д2 }}+\mathrm{Z}_{\text {д3 }}+\mathrm{Z}_{\text {д4 }}+\mathrm{Z}_{\text {д5}} .
$$

Относительная площадь поперечного сечения бруса

$$
Z_{\text {бр }}=\mathrm{m}_{\mathrm{H}} 4 \mathrm{~m}_{\mathrm{A}} \text {. }
$$

Суммарная относительная площадь поперечных сечений бруса и боковых обрезных досок

$$
Z=Z_{\text {бр }}+Z_{\text {д }} \text {. }
$$

При дальнейшем раскрое бруса (второй проход) получаются обрезные доски, а также образуются потери в виде опилок. Объем опилок зависит от числа выпиливаемых досок из бруса. Количество пропилов при раскрое бруса на обрезные доски может быть различным. Если боковые пропилы находятся (расположены) вне пласти бруса, то количество учитываемых пропилов следует определять по формуле

$$
\mathrm{i}_{1}=\mathrm{n}-1
$$

где $\mathrm{n}$ - число обрезных досок, выпиливаемых из бруса.

Если один из пропилов расположен в зоне пласти бруса, то количество учитываемых пропилов необходимо определять по выражению

$$
\mathrm{i}_{2}=\mathrm{n} \text {. }
$$

Если два боковых пропила расположены в зоне пласти бруса, то количество учитываемых пропилов необходимо определять по выражению

$$
\mathrm{i}_{3}=\mathrm{n}+1 \text {. }
$$

Число досок получаемых из бруса может находится, как правило, в пределах 12-20 в зависимости от диаметра пиловочника и толщины получаемых обрезных досок из бруса. Тогда потери древесины в опилкипри раскрое бруса (площадь поперечного сечения пропилов) можно определить по формуле

$$
\mathrm{Z}_{\text {пр }}=\mathrm{i} \times \mathrm{m}_{\mathrm{H}} \times \mathrm{m}_{\mathrm{e}} .
$$

С учетом потерь древесины в опилки при распиловке бруса площадь поперечного сечения получаемых обрезных досок из него определится по формуле

$$
Z_{\text {д бр }}=Z_{\text {бр }}-Z_{\text {пр }} \text {. }
$$

С учетом потерь древесины в опилки, получаемых при раскрое бруса, объем обрезных досок определится по формуле

$$
\mathrm{Z}=\mathrm{Z}_{\text {д }}+\mathrm{Z}_{\text {д } 6 \mathrm{p}} \text {. }
$$

Результаты расчетов

В расчетах принимаем следующие варианты: $\mathrm{i}=0, i=4, i=8, i=12, i=16, i=20$.

Расчеты производятся следующим образом. Прежде всего принимаем число пропилов і и для этого варианта выполняются расчеты. Вначале задаем относительную ширину пропила, а затем относительную толщину бруса в предполагаемом диапазоне 0,2-0,4 с градацией 0,05 и определяем все размеры бруса и досок, а также величину целевой функции. По результатам расчетов находим диапазон $\mathrm{m}_{\mathrm{H}}$, в котором целевая функция принимает максимальное значение. Далее для этого диапа- 


\section{Деревопереработка. Химические технологии}

зона производим расчеты с градацией $\mathrm{m}_{\mathrm{H}}=0,01$ и определяем все размеры бруса и досок, а также величину целевой функции. Затем по результатам расчетов находим диапазон $\mathrm{m}_{\mathrm{H}}$, в котором целевая функция принимает максимальное значение. Далее для этого диапазона окончательно производим расчеты при изменении $\mathrm{m}_{\mathrm{H}}$ с градацией 0,001 и определяем максимальное значение целевой функции.
Этот результат принимаем за искомый вариант решения задачи, так как такая точность расчета вполне достаточна. Результаты расчетов записываем в табл. 1.

Результаты расчетов показывают, что с увеличением ширины пропила при первом проходе раскроя бревна толщина бруса возрастает, а разме-

Таблица 1

Оптимальные относительные размеры бруса и досок для различного количества пропилов при разной относительной ширине пропила

\begin{tabular}{|c|c|c|c|c|c|c|c|c|c|c|c|c|c|c|c|c|}
\hline $\mathrm{i}$ & $\mathrm{m}_{\mathrm{e}}$ & $\mathrm{m}_{\mathrm{H}}$ & $\mathrm{m}_{\mathrm{A}}$ & $\mathrm{m}_{\mathrm{b} 1}$ & $\mathrm{~m}_{\mathrm{T} 1}$ & $\mathrm{~m}_{\mathrm{b} 2}$ & $\mathrm{~m}_{\mathrm{T} 2}$ & $\mathrm{~m}_{\mathrm{b} 3}$ & $\mathrm{~m}_{\mathrm{T} 3}$ & $\mathrm{~m}_{\mathrm{b4}}$ & $\mathrm{m}_{\mathrm{T} 4}$ & $\mathrm{~m}_{\mathrm{T} 5}$ & $\mathrm{~m}_{\mathrm{b} 5}$ & $Z_{\text {дбр }}$ & $Z_{\text {д }}$ & Z \\
\hline 0 & 0 & 0,28 & 0,96 & 0,878 & 0,099 & 0,771 & 0,080 & 0,639 & 0,066 & 0,480 & 0,054 & 0,041 & 0,282 & 0,2688 & 0,4560 & 0,7248 \\
\hline 0 & 0,002 & 29 & 0,957 & 869 & 0,100 & .755 & 0,079 &, 619 & 0,063 &, 458 & 0,049 & 0,036 & 0,267 & 0,2775 &, 4353 &, 7128 \\
\hline 0 & 0,004 & 0,3 & 0,954 & 0,860 & 0,101 & 0739 & 0,077 & 0 & 0,060 & 0,437 & 0,045 & 0,030 & 0,252 & 0,2862 & 0,4150 & 0,7011 \\
\hline 0 & 006 & 0,31 & 0,951 & 850 & 0,103 & ,722 & 0,076 & 0,576 & 0,057 &, 415 & 0,040 & 0,025 & , 239 & 0,2947 &, 3951 & 0,6899 \\
\hline 0 & 0,008 & 0,33 & 0,944 & 0,829 & 0,107 & 0,684 & 0,077 & 0,021 & 0,054 & 0243 & 0,035 & 0,016 & 0,151 & 0,3115 & 0,3675 & 0,6791 \\
\hline 0 & 0,01 & 0,34 & 0,940 & 0,818 & 0,108 & 0,665 & 0,075 & 0,496 & 0,051 & 0,319 & 0,030 & 0,011 & 0,141 & 0,3197 & 0,3493 & 0,6691 \\
\hline 4 & 0,002 & 0,29 & 0,957 & ,869 & 0,100 & ,755 & 0,079 & 0,619 & 0,063 & 0,458 & 0,049 & 0,036 & 0,267 & 0,2752 & 0,4353 &, 7105 \\
\hline 4 & 004 & 0,3 & 0,954 & 0,860 & 0,101 & 0730 & 0,077 & טרנס, & 0,060 & 437 & 0,045 & 0,030 & 0,252 & 0,2814 & 0,4150 & 0,6963 \\
\hline 4 & 006 & 0,31 & 0,951 & 850 & 0,103 & 722 & 0,076 & ,576 & 0,057 &, 41 & 0,040 & 0,02 & ,23 & 2873 & 3951 & 0,6824 \\
\hline 4 & 0,008 & 0,32 & 0,947 & 0,839 & 0,104 & 0,705 & 0,075 & 0,534 & 0,054 & נינכ, & 0,035 & 0,019 & 0,228 & 0,2929 & 0,3758 & 0,6687 \\
\hline 4 & 0,01 & 0,34 & 0,940 & 0,818 & 0,108 & 0,665 & 0,075 & 0,496 & 0,051 & 0319 & 0,030 & 0,011 & 0,141 & 0,3061 & 3493 & 0,6555 \\
\hline 8 & 0,002 & 0,29 & 0,957 & 0,869 & 0,100 & 0,755 & 0,079 & 0,619 & 0,063 & 0,458 & 0,049 & 0,036 & 0,267 & 0,2729 & 0,4353 & 0,7081 \\
\hline 8 & 0,004 & 0,3 & 0,954 & 0,860 & 0,101 & 0,739 & 0,077 & 0 & 0,060 & 0 & 0,045 & 0,030 & 0,252 & & & 0,6915 \\
\hline 8 & 0,006 & 0,31 & 0,951 & 0,850 & 0,103 & 0,722 & 0,076 & 0,576 & 0,057 & 5 & 0,040 & & & & & 0,6750 \\
\hline 8 & 0,008 & 0,32 & 0,947 & 0,839 & 0,104 & 0,705 & 0,075 & 0,534 & 0,054 & 0,393 & 0,035 & 0,019 & 0,228 & 0,2827 & 0,3758 & 0,6585 \\
\hline 8 & 0,01 & 0,34 & 0,940 & 0,818 & 0,108 & 0,665 & 0,075 & 0,496 & 0,051 & 0,319 & 0,030 & 0,011 &, 141 & 2925, & 0,3493 & 0,6419 \\
\hline 12 & 0,002 & 0,29 & 0,957 & 0,869 & 0,100 & 0,755 & 0,079 & 0,619 & 0,063 & 0,458 & 0,049 & 0,036 & 0,267 & 0,2706 & 0,4353 & 0,7058 \\
\hline 12 & 0,004 & 0,3 & 0,954 & 0,860 & 0,101 & 0,739 & 0,077 & 0,390 & 0,060 & T & 0,043 & 0,030 & 0,252 & 0,2718 & 0,4150 & 0,6867 \\
\hline 12 & 0,006 & 0,31 & 0,951 & 0,850 & 0,103 & 0,722 & 0,076 & 0,576 & 0,057 & 0,415 & 0,040 & 0,025 & 0,239 & & 0,3951 & 0,6675 \\
\hline 12 & 0,008 & 0,32 & 0,947 & 0,839 & 0,104 & 0,705 & 0,075 & 0,554 & 0,054 &, 393 & 0,035 & 0,019 & 0,228 & ,2725 & 0,3758 & 0,6482 \\
\hline 12 & 0,01 & 0,33 & 0,944 & 0,829 & 0,105 & 0,687 & 0,073 & 0,531 & 0,050 & 0,371 & 0,031 & 0,014 & 0,218 & & & \\
\hline 16 & 0,002 & 0,29 & 0,957 & 0,869 & 0,100 & 0,755 & 0,079 & 0,619 & 0,063 & 0,458 & 0,049 & 0,036 & 0,267 & & 0,4353 & 0,7035 \\
\hline 16 & 0,004 & 0,3 & 0,954 & 0,860 & 0,101 & 0,739 & 0,077 & 0,598 & 0,060 & 0,457 & 0,045 & 0,030 & 0,252 & 0,2670 & 0,4150 & 0,6819 \\
\hline 16 & 0,006 & 0,31 & 0,951 & 0,850 & 0,103 & 0,722 & 0,076 & 0,576 & 0,057 & 0,415 & 0,040 & 0,025 & 0,239 & 0,2650 & 0,3951 & 0,6601 \\
\hline 16 & 0,008 & 0,32 & 0,947 & 0,839 & 0,104 & 0,705 & 0,075 & 0,554 & 0,054 & 0,393 & 0,035 & 0,019 & 0,228 & 0,2622 & 0,3758 & 0,6380 \\
\hline 16 & 0,01 & 0,33 & 0,944 & 0,829 & 0,105 & 0,687 & 0,073 & 0,531 & 0,050 & 0,371 & 0,031 & 0,014 & 0,218 & 0,2587 & 0,3569 & 0,6156 \\
\hline 20 & 0,002 & 0,29 & 0,957 & 0,869 & 0,100 & 0,755 & 0,079 & 0,619 & 0,063 & 0,458 & 0,049 & 0,036 & 0,267 & 0,2659 & 0,4353 & 0,7012 \\
\hline 20 & 0,004 & 0,3 & 0,954 & 0,860 & 0,101 & 0,739 & 0,077 & 0,598 & 0,060 & 0,437 & 0,045 & 0,030 & 0,252 & 0,2622 & 0,4150 & 0,6771 \\
\hline 20 & 0,006 & 0,31 & 0,951 & 0,850 & 0,103 & 0,722 & 0,076 & 0,576 & 0,057 & 0,415 & 0,040 & 0,025 & 0,239 & 0,2575 & 0,3951 & 0,6527 \\
\hline 20 & 0,008 & 0,32 & 0,947 & 0,839 & 0,104 & 0,705 & 0,075 & 0,554 & 0,054 & 0,393 & 0,035 & 0,019 & 0,228 & 0,2520 & 0,3758 & 0,6278 \\
\hline 20 & 0,01 & 0,33 & 0,944 & 0,829 & 0,105 & 0,687 & 0,073 & 0,531 & 0,050 & 0,371 & 0,031 & 0,014 & 0,218 & 0,2455 & 0,3569 & 0,6024 \\
\hline
\end{tabular}


ры боковых обрезных досок уменьшаются (рис. 2).Относительная ширина крайней боковой доски при увеличении относительной ширины пропила до 0,01 уменьшается примерно в 2 раза (рис. 2). При этом относительная толщина второй боковой доски от края пиловочника при увеличении относительной ширины пропила до 0,01 уменьшается всего лишь в 1,5 раза.

Оптимальные относительные толщины боковых досок изменяются с увеличением относительной ширины пропила своеобразно (рис. 3). Относительная толщина первой пары боковых досок возрастает, а относительные толщины остальных досок уменьшаются. Причем, размеры крайних боковых досок уменьшаются в большей мере, чем боковые доски, расположенные ближе к середине пиловочника (рис. 3). Относительная толщина последней крайней боковой доски при увеличении относительной ширины пропила до 0,01 уменьшается в 3,7 раза по сравнению с относительной толщиной этой доски при $\mathrm{m}_{\mathrm{e}}=0$. При этом относительная толщина второй боковой доски при $\mathrm{m}_{\mathrm{e}}=0,01$ уменьшается всего лишь в 1,07 раза по сравнению с относительной толщиной этой доски при $\mathrm{m}_{\mathrm{e}}=0$.

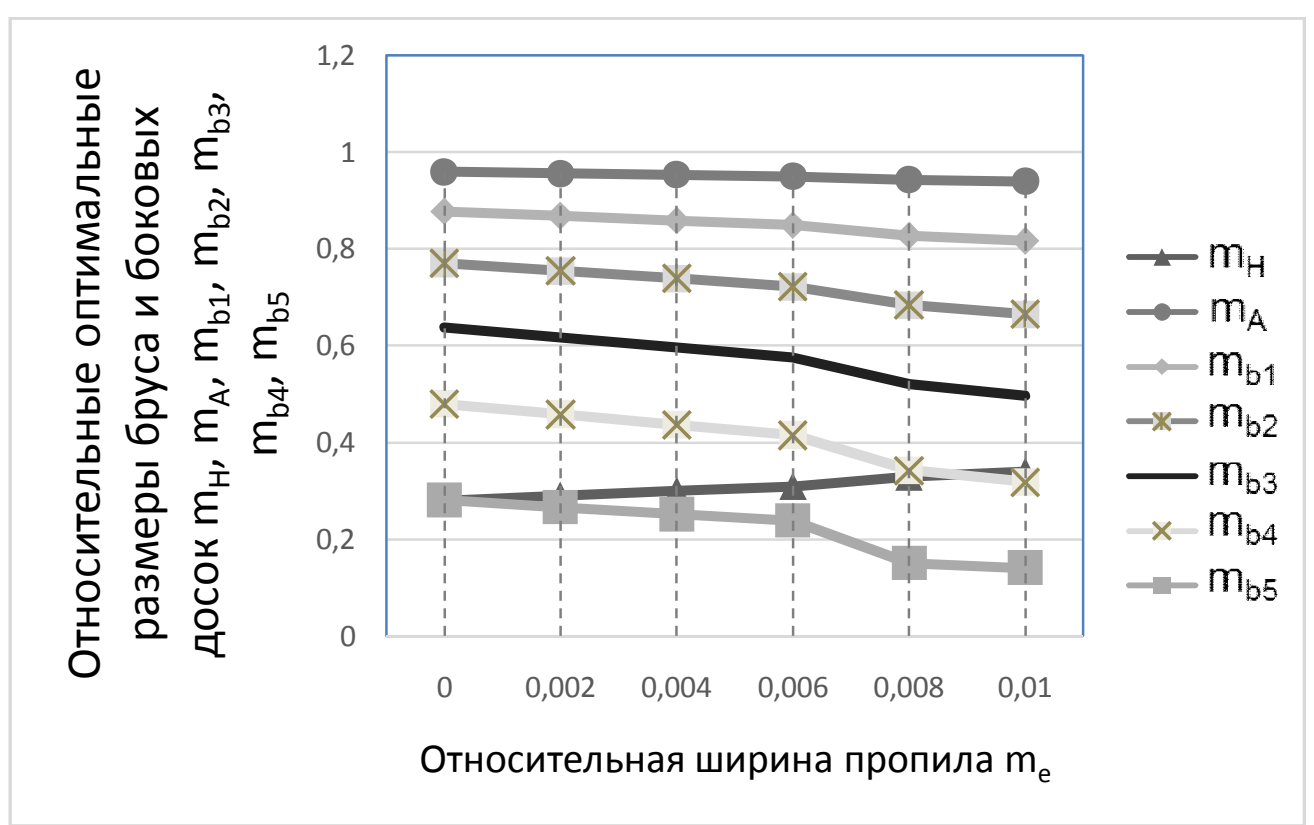

Рис. 2. Влияние относительной ширины пропила на оптимальные относительные размеры бруса и относительные ширины боковых обрезных досок при $\mathrm{i}=0$

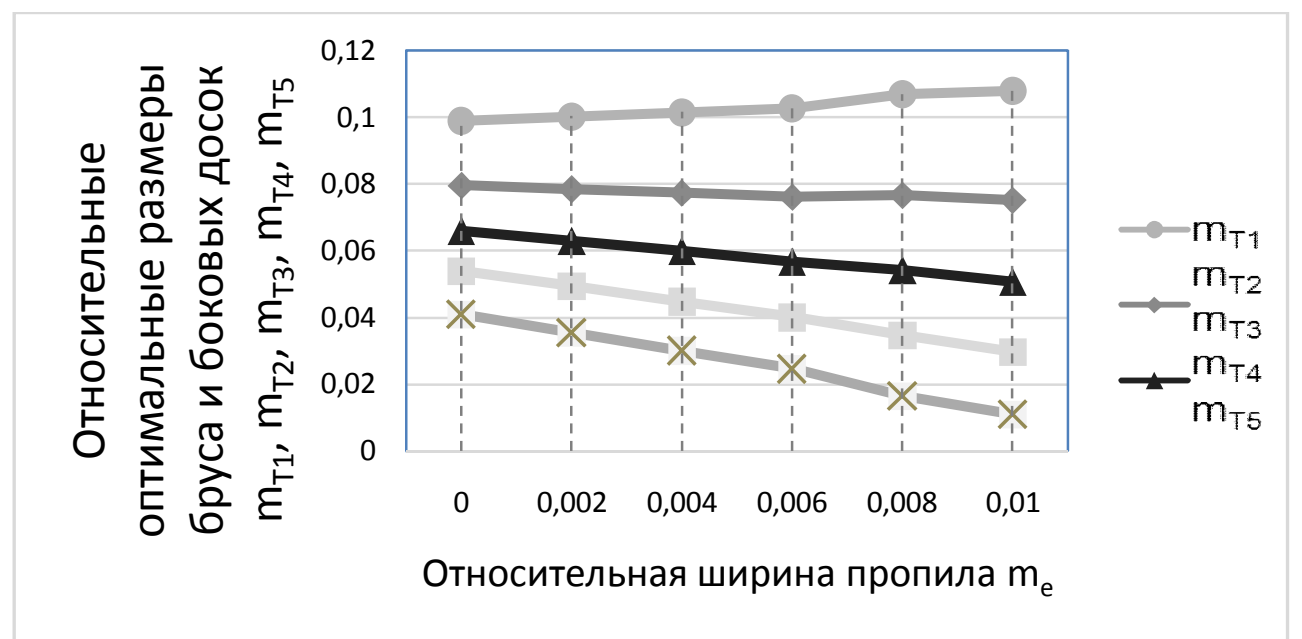

Рис. 3. Влияние относительной ширины пропила на оптимальные относительные толщины боковых обрезных досок при $\mathrm{i}=0$ 


\section{Деревопереработка. Химические технологии}

С увеличением относительной ширины пропила относительная площадь поперечного сечения бруса возрастает, а относительная площадь поперечного сечения боковых обрезных досок уменьшается (рис. 4). При этом величина целевой функции уменьшается. Следовательно, уменьшение площади поперечного сечения боковых досок происходит в большей степени, чем увеличение площади поперечного сечения бруса.

При раскрое бруса на обрезные доски оптимальные относительные размеры бруса и боковых обрезных досок существенно не изменяются. При этом важно знать, каким образом оказывает влияние количество пропилов на оптимальные относительные размеры боковых обрезных досок при распиловке бруса на обрезные доски. С увеличением количества пропилов относительная оптимальная толщина бруса практически не изменяется, а относительные ширины боковых обрезных досок сохраняют прежнее значение (рис. 5).

С увеличением количества пропилов при распиловке бруса на обрезные доски относительные оптимальные толщины боковых обрезных досок не изменяются (рис. 6).

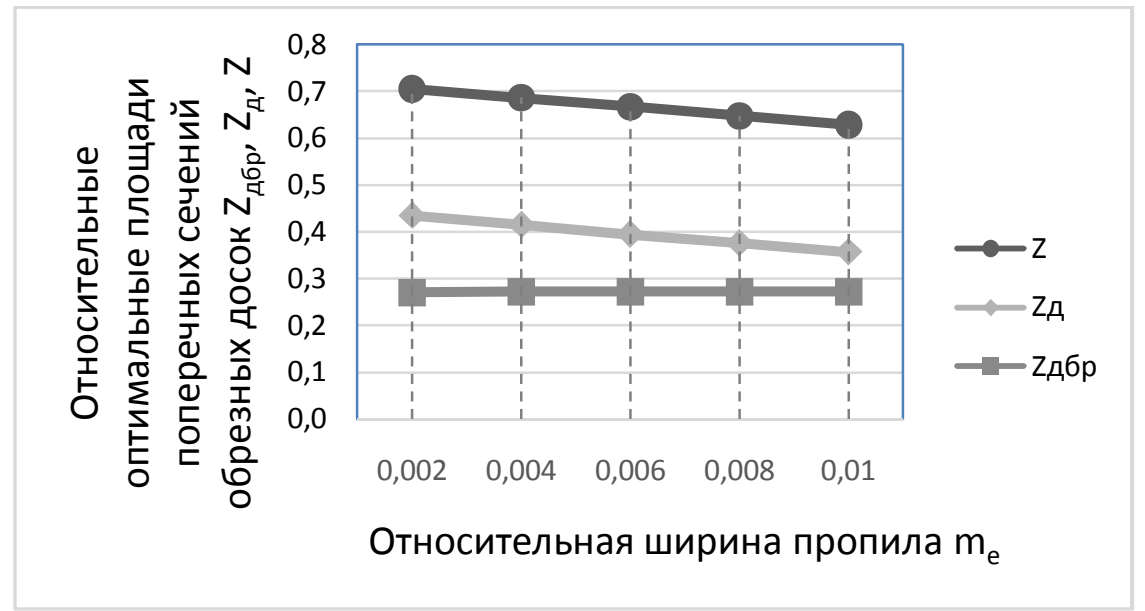

Рис. 4. Влияние относительной ширины пропила на оптимальные относительные площади поперечных сечений бруса и боковых обрезных досок при $\mathrm{i}=0$

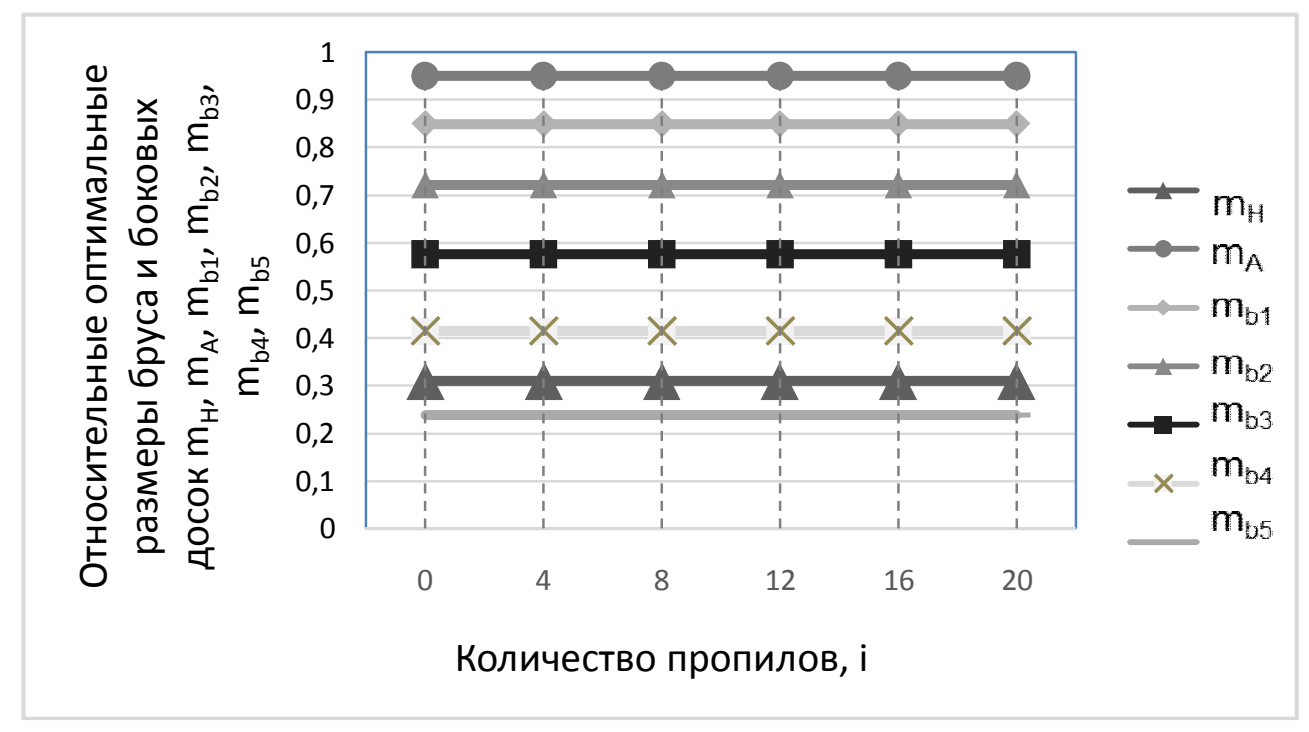

Рис. 5. Влияние количество пропилов при распиловке бруса на оптимальные относительные ширины обрезных досок при $\mathrm{m}_{\mathrm{e}}=0,006$ 


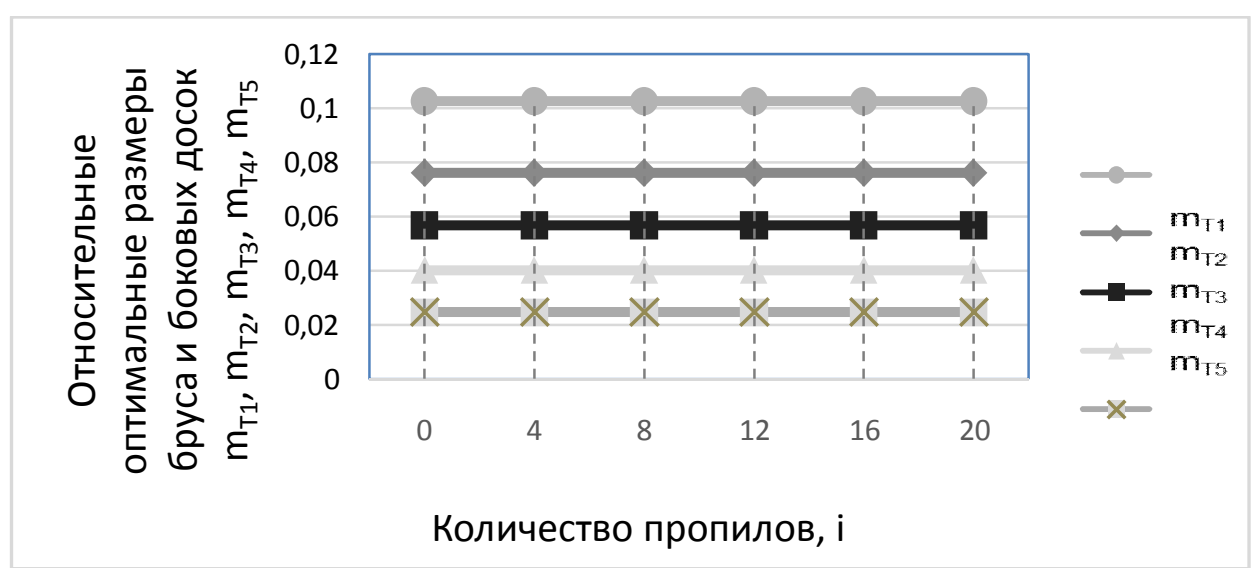

Рис. 6. Влияние количество пропилов на оптимальные относительные толщины боковых обрезных досок при $\mathrm{m}_{\mathrm{e}}=0,006$

С увеличением количества пропилов при распиловке брусьев на обрезные доски площади поперечных сечений досок, получаемых из бруса,незначительно уменьшаются, а относительные оптимальные площади поперечных сечений боковых обрезных досок сохраняют прежнее значение (рис. 7). В итоге величина целевой функции с увеличением количества пропилов при распиловке бруса на обрезные доски уменьшается. Следовательно, величина целевой функции изменяется за счет изменения площади поперечных сечений обрезных досок, получаемых из бруса.

Расчеты показывают, что при относительной ширине пропила $\mathrm{m}_{\mathrm{e}}=0,006$ толщина пятой пары боковых обрезных досок составляет всего лишь 0,025 от диаметра бревна в вершинном торце. Следовательно, выпиливать пятую пару боковых об- резных досок для такой схемы раскроя не всегдацелесообразно, так как получаются по расчету очень тонкие доски. Толщина первой пары боковых обрезных досок составляет 0,10 от диаметра пиловочника в вершинном торце, что вполне можно реализовать на практике.

Анализируя результаты расчетов следует отметить, что при раскрое двухкантного бруса с числом пропилов $\mathrm{i}=12 \ldots 16$ и относительной ширине пропила $\mathrm{m}_{\mathrm{e}}=0,01 \ldots 0,006$ оптимальная относительная толщина этого бруса изменяется в пределах $\mathrm{m}_{\mathrm{H}}=0,31 \ldots 0,33$ и в среднем составляет 0,32 от диаметра бревна в вершинном торце.

\section{Выводы и рекомендации}

Решена задача оптимизации раскроя пиловочника больших и крупных размеров с получением бруса и пяти пар боковых досок с учетом шири-

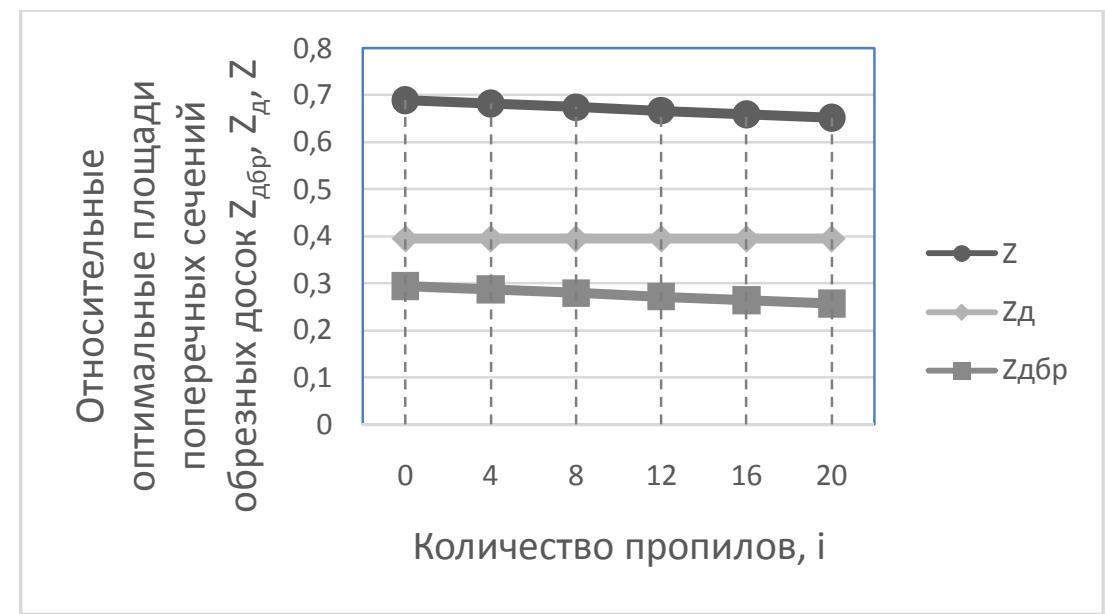

Рис. 7. Влияние количества пропилов на оптимальные относительные площади поперечных сечений бруса и досок при $\mathrm{m}_{\mathrm{e}}=0,006$ 


\section{Деревопереработка. Химические технологии}

ны пропила и количества пропилов при раскрое бруса на обрезные доски. Составлена математическая модель данной схемы раскроя пиловочника. Численным методом получены оптимальные относительные размеры бруса и досок в зависимости от ширины пропила, которые рекомендуется использовать при выборе варианта постава. Подтверждается ранее принятая гипотеза о том, что с увеличением объема бруса, объем боковых досок уменьшается и наоборот. Таким образом имеется такое соотношение размеров бруса и боковых досок, при котором объем этой пилопродукции получается максимальным. С увеличением ширины пропила, толщина бруса возрастает, а размеры боковых досок уменьшаются. При раскрое бруса на обрезные доски с увеличением количества пропилов оптимальная толщина бруса не изменяется, а оптимальные размеры боковых досок сохраняют свои размеры.

Таким образом, ширина пропила оказывает влияние на оптимальные размеры бруса в большей мере, чем количество пропилов при распиловке бруса на обрезные доски. Алгоритм решения задачи рекомендуется использовать при расчете и составлении поставов, а также для разработки программ расчета поставов на ЭВМ.

\section{Библиографический список}

1. Агапов, А. И. Оптимизация раскроя пиловочника средних размеров при брусово-развальном способе распиловки [Текст] / А. И. Агапов // Современная наука : актуальные проблемы и пути их решения : 7-я междунар. дистанционная науч. конф., Липецк, 20-21 февраля 2014 г. - Липецк : МАКСИМАЛ, 2014. - С. 16-24.

2. Агапов, А. И. Оптимизация раскроя пиловочника с выпиливанием трех брусьев разной толщины и двух пар боковых досок [Текст] / А. И. Агапов // Актуальные проблемы развития лесного комплекса: междунар. научно-технич. конф., Вологда, 3-4 декабря 2013 г. - Вологда, 2014. - С. 62-66.

3. Агапов, А. И. Влияние ширины пропила на оптимальные размеры брусьев и досок при раскрое пиловочника с выпиливанием трёх брусьев одинаковой толщины и четырёх пар боковых досок [Электронный ресурс] / А. И. Агапов // Лесотехнический журнал. - 2014. - Т. 4. - № 2. - С. 128-135.

4. Аксенов, П. П. Теоретические основы раскроя пиловочного сырья [Текст] / П. П. Аксенов. М. : Лесн. пром-сть, 1980. - 216 с.

5. Ветшева, В. Ф. Раскрой крупномерных бревен на пиломатериалы [Текст] / В. Ф. Ветшева. М. : Лесн. пром-сть, 1976. - 168 с.

6. Виллистон, Э. Производство пиломатериалов [Текст] / Э. Виллистон. - М. : Лесн. пром-сть, 1981. -384 с.

7. Власов, Г. Д. Новые методы технологических расчетов в лесопилении [Текст] / Г. Д. Власов. М. ; Л. : Гослесбумиздат, 1954. - 60 с.

8. Калитеевский, Р. Е. Лесопиление в XXI веке. Технология, оборудование, менеджмент [Текст] / Р. Е. Калитеевский. - Изд. 2-е, испр. и доп. - СПб. : Профи КС, 2008. - 496 с.

9. Калитеевский, Р. Е. Проектирование лесопильных потоков [Текст] / Р. Е. Калитеевский. - М. : Лесн. пром-сть, 1972. $-184 \mathrm{c}$.

10. Рыкунин, С. Н. Планирование раскроя сырья методом линейного программирования [Текст] / С. Н. Рыкунин. - М. : Лесн. пром-сть, 1965. - № 8. - С. 25-27.

11. Соболев, И. В. Оптимизация раскроя пиловочного сырья [Текст] / И. В. Соболев. - М. : Лесн. пром-сть, 1971. $-36 \mathrm{c}$.

12. Стоевюь Г. И. Определение максимального выхода пиломатериалов [Текст] / Г. И. Стоев. М. : Лесн. пром-сть, 1961. - 64 с.

13. Уласовец, В. Г. Технологические основы производства пиломатериалов [Текст] / В. Г. Уласовец. Екатеринбург, 2002. - 510 с.

14. Combined production of sawn timber and firewood billets at a birch sawmill in Finland : a simulation approach [Text] / T. Tahvanainen [et al.] // Wood Material Science \& Engineering. - 2008. - V. 3. - I. 1-2. - Pp. 1-11.

15. Gil, A. Log classification in the hardwood timber industry [Text] : method and value analysis / A. Gil, J.-M. Frayret // International Journal of Production Research. - 2016. - Vol. 54. - I. 15. - Pp. 4669-4688. 
16. Pilkerton, S. J. Development of efficient cutting patterns to maximize value with a log-allocation constraint [Text] / S. J. Pilkerton, J. Sessions, L. D. Kellog // International Journal of Forest Engineering. - 2013. - Vol. 24. - I. 1. - Pp. 42-52.

17. Product potential of paulownia timber [Text] / M. Beel [et al.] // Australian Forestry. - 2005. - Vol. 68. - I. 1. - Pp. 3-8.

18. Todoroki, C. Accuracy considerations when optimally sawing pruned logs : internal defects and sawing precision [Text] / C. Todoroki // Nondestructive Testing and Evaluation. - 2003. - Vol. 19. - I. 1-2. - Pp. 29-41.

19. Todoroki, C. Dynamic control of timber production at a sawmill with log sawing optimization [Text] / C. Todoroki, M. Rönnqvist // Scandinavian Journal of Forest Research. - 2002. - Vol. 17. - I. 1. - Pp. 79-89.

\section{References}

1. Agapov A. I. Optimizaciya raskroya pilovochnika srednih razmerov pri brusovo_razvalnom sposobe raspilovki [Optimization of cutting logs with a medium-sized lumber-cutting method breakup] Sovremennaya nauka_aktualnie problemi i puti ih resheniya: 7-ya mejdunar. distancionnaya nauch. konf. [Modern science : actual problems and ways of their solution : 7-I-Nar. remote scientific. Conf.]. Lipeck, 2014, pp. 16 (In Russian).

2. Agapov A. I. Optimizaciya raskroya pilovochnika s vipilivaniem treh brusev raznoi tolschini i dvuh par bokovih dosok [Optimization of cutting logs with sawing out three beams of different thicknesses and two pairs of side boards] Aktualnie problemi razvitiya lesnogo kompleksa_mejdunar. nauchno_tehnich. konf. [Actual problems of forest sector development: Intern. nauchno-tekhnich. Conf]. Vologda, 2014, pp. 62-66. (In Russian).

3. Agapov A. I. Vliyanie shirini propila na optimalnie razmeri brusev $i$ dosok pri raskroe pilovochnika s vipilivaniem treh brusev odinakovoi tolschini $i$ chetireh par bokovih dosok [Influence of the width of cut on the optimum size of the boards and the boards when cutting logs with three sawing boards of the same thickness, and four pairs of side boards] Lesotehnicheskii jurnal, 2014, Vol. 4, no. 2, pp. 128-135. (In Russian).

4. Aksenov P. P. Teoreticheskie osnovi raskroya pilovochnogo sirya [Theoretical Foundations of cutting sawlogs]. Moscow, 1980, 216 p. (In Russian).

5. Vetsheva V. F. Raskroi krupnomernih breven na pilomateriali. [Cutting large-sized logs for lumber] Moscow, 1976, 168 p. (In Russian).

6. Villiston E. Proizvodstvo pilomaterialov [Production of sawn timber]. Moscow, 1981, 384 p. (In Russian).

7. Vlasov G. D. Novie metodi tehnologicheskih raschetov v lesopilenii [New methods of technological calculations in the sawmill]. Moscow, 1954, 60 p. (In Russian).

8. Kaliteevskii R. E. Lesopilenie v 21 veke. Tehnologiya_oborudovanie_menedjment.[Lumbering in the 21 century. Technology, equipment, management.] Saint Petersburg, 2008, 496 p. (In Russian).

9. Kaliteevskii R. E. Proektirovanie lesopilnih potokov [Design flows sawmills]. Moscow, 1972, 184 p. (In Russian).

10. Rikunin S. N. Planirovanie raskroya sirya metodom lineinogo programmirovaniya [Raw material cutting planning using linear programming]. Moscow, 1965, no. 8, pp.25-27. (In Russian).

11. Sobolev I. V. Optimizaciya raskroya pilovochnogo sirya [Optimization of cutting sawlogs]. Moscow, 1971, 36 p. (In Russian).

12. Stoev G. I. Opredelenie maksimalnogo vihoda pilomaterialov [Determination of the maximum output of sawn timber]. Moscow, 1961, 64 p. (In Russian).

13. Ulasovec V. G. Tehnologicheskie osnovi proizvodstva pilomaterialov [Technological bases of lumber production]. Ekaterinburg, 2002, 510 p. (In Russian).

14. Tahvanainen T. [et al.] Combined production of sawn timber and firewood billets at a birch sawmill in Finland Wood Material Science \& Engineering, 2008, Vol. 3, Issue. 1-2, pp. 1-11.

15. Gil A., Frayret J.-M. Log classification in the hardwood timber industry: method and value analysis International Journal of Production Research, 2016, Vol. 54, I. 15, pp. 4669-4688.

16. Pilkerton S. J., Sessions J., Kellog L. D. Development of efficient cutting patterns to maximize value with a logallocation constraint International Journal of Forest Engineering, 2013, Vol. 24, I. 1, pp. 42-52.

17. Beel M. [et al.] Product potential of paulownia timber. Australian Forestry, 2005, Vol. 68, I. 1, pp. 3-8. 


\section{Деревопереработка. Химические технологии}

18. Todoroki C. Accuracy considerations when optimally sawing pruned logs : internal defects and sawing precision Nondestructive Testing and Evaluation, 2003, Vol. 19, I. 1-2, pp. 29-41.

19. Todoroki C., Rönnqvist M. Dynamic control of timber production at a sawmill with log sawing optimization Scandinavian Journal of Forest Research, 2002, Vol. 17, I. 1, pp. 79-89.

\section{Сведения об авторе}

Агапов Александр Иванович - профессор кафедры механической технологии древесины ФГБОУ ВО «Вятский государственный университет», доктор технических наук, профессор, г. Киров, Российская Федерация; e-mail: kaf_mtd@vyatsu.ru.

\section{Information about author}

Agapov Aleksandr Ivanovich - Professor of Department of mechanical wood technology Federal State Budget Education Institution of Higher Education «Vyatka State University», DSc in Engineering, Professor, Kirov, Russian Federation; e-mail:kaf_mtd@vyatsu.ru.

DOI: $12737 / 25205$

УДК 674.815-41

\section{ВЛИЯНИЕ СОВРЕМЕННЫХ ТЕХНОЛОГИЙ НА ТРЕБОВАНИЯ СТАНДАРТА К КАЧЕСТВУ ДРЕВЕСНО-СТРУЖЕЧНЫХ ПЛИТ}

кандидат технических наук, доцент Е. В. Кантиева ${ }^{1}$

кандидат технических наук, доцент Л. В. Пономаренко ${ }^{1}$

А. Э. Черняев ${ }^{1}$

1- ФГБОУ ВО «Воронежский государственный лесотехнический университет им. Г.Ф. Морозова»,

г. Воронеж, Российская Федерация

Понятие качества в сознании советского и российского человека неразрывно связано с понятием ГОСТ. Предъявляемые стандартом требования к качеству продукции не остаются постоянными, а меняются в соответствии с уровнем развития науки, техники и передовым опытом. В статье рассматривается динамика изменений требований ГОСТ 10632 «Плиты древесностружечные. Технические условия» к качеству древесно-стружечных плит за последние 40 лет. С этой целью проанализированы редакции данного ГОСТ, утвержденные в 1977 , 1989, 2007 и 2014 гг. За указанный период времени изменилась маркировка плит с П-А и П-Б на Р1, Р2, что связано с гармонизацией с зарубежными стандартами. В последней редакции ГОСТ 2014 года плиты не имеют ограничений по размерам, так как на смену прессам периодического действия, имеющих определенные размеры нагревательных плит пресса, пришли прессы непрерывного прессования. Такая же ситуация и с плотностью плит, производитель сам устанавливает ее значение, но при этом прочность плит должна соответствовать требованиям стандарта. Хотя прочностные показатели (предел прочности при растяжении перпендикулярно пласти и предел прочности при статическом изгибе) с каждой новой редакцией ГОСТ уменьшаются. Ужесточение требований произошло к санитарно-гигиеническим показателям древесно-стружечных плит. Содержание свободного формальдегида снизилось с 10 до 4 мг на 100 г абсолютно сухой плиты. Для получения конкурентоспособных плит класса эмиссии $\mathrm{E}_{0}$ российской химической промышленности необходимо разработать большой объем смол с низким содержанием свободного формальдегида.

Ключевые слова: стандарт, качество, древесно-стружечная плита, показатель. 